\title{
Committed to progress of endocrinology \& metabolism international journal
}

\section{Editorial}

On the Advent of the New Year 2016, while we bid farewell year 2015, we have concluded our achievements by launching the last issue for 2015 of Endocrine and Metabolism International journal (EMIJ) as scholarly peer-review imminent journal for health researchers and professionals involved in the care of people with Endocrine, Metabolic and Diabetes diseases.

EMIJ that aims to publish the most complete and reliable source of information on the discoveries and current developments in the form of original articles, review articles, case reports etc. aiming for improving professional standard in both research on one hand and clinical practice on the other, seeking high quality and increased credibility .Our vision is to share new research and clinical knowledge in diabetes and endocrine diseases with the health professional community, so that ultimately, our patients can have better care and quality of life EMIJ offers an (Open Access) platform to the authors to publish their research outcome, we plan to make this task; readily available, feasible and transparent, in order to involve all the professionals around the globe in accomplishing our mission...

Each year, new knowledge and treatments for diabetes and endocrine diseases are uncovered in many parts of the world.

The increase in knowledge of the subject of endocrinology, like that of so many other branches of medicine, has been so rapid in the past decade or two that it is most difficult, even for one professedly engaged in the specialty, to keep abreast of all the advances made.

an enormous literature on the thyroid gland has developed within the past few years demonstrated appreciable advances in clinical knowledge, especially relating to diagnosis and treatment of thyroid disorders, there have been developments of fundamental importance in the physiology of thyroid function, some aspects of the adrenal cortex; pituitary insufficiency in the adult; and some recent developments in our knowledge of intersexuality, Vitamin D and bone biology, improvement in treatment of osteoporosis, more understanding about the knowledge and management of ovarian problems, like PCOS, more global interest on reviewing hormonal therapy and addressing more refined indications and safety. There are more understanding on molecular structure of receptors and scientific implementation in treatment of pituitary and adrenal neoplasms.

Among other endocrine disorders, genomics will unquestionably enhance our understanding and management of disease. The incidence of thyroid cancer has been increasing steadily in 2014, it was estimated there were 62,980 new cases of thyroid cancer in the US alone. New knowledge in molecular diagnosis of thyroid cancer may greatly influence the choice of treatments and would benefit those affected. In adrenocortical carcinoma, genomic studies have allowed us to distinguish two distinctive groups of patients; one with poor prognosis and another with a far more favorable prognosis...
Volume 2 Issue 4 - 2015

\author{
Saadi AlJadir \\ Ministry of Higher Education, Iraq
}

Correspondence: Saadi AlJadir, Ministry of Higher Education, P.O. Box 498, Nassiriya, Thi Qar, Iraq, Tel +96478I3902926, Email saljadir5I@gmail.com

Received: November 30, 2015 | Published: December 07, 2015

In diabetes, discovery and development of the 11 classes of antihyperglycemia medications available today has spanned over nine decades, and still many are yet evolving in the pipeline...

These medications have helped to improve glycemic control, better quality of life and fewer disabilities, but we need continued advances in understanding diabetes so that the burden of disease can be decreased both for individual patients and for society as a whole.

From ideas, guidelines, consensuses to patients, translational researches span the spectra from basic science, to clinical research, to clinical trials, to approval by regulatory agencies. Because as many as 387 million patients worldwide have been diagnosed with diabetes in 2013 and this number might be doubled in the forthcoming 2 decades!

Therefore it is imperative for researches and professionals to have progress in all these aspects...

In last few years there was impressive eruption of new long acting insulins (Analogs), with better biological properties and better glycemic control, new class of oral medications utilizing the kidney role in glucose homeostasis; Sodium glucose co-transporter inhibitors, adding effective class of medication to armamentarium of diabetes control.

There notably are an increasing interest and rather restrictive rules of drugs approval agencies (e.g. FDA) on drug safety and cardiovascular impact. The academic health institutions have revolutionized their guidelines, pioneered presence at recognizable platforms of global meetings for updating and refining their addressed guidelines and consensus.

\section{Acknowledgements}

None.

\section{Conflicts of interest}

The author declares there is no conflict of interest. 